\title{
Convex Independent Sets and 7-Holes in Restricted Planar Point Sets
}

\author{
Pavel Valtr \\ Department of Applied Mathematics, Charles University, \\ Malostranské nám. 25, 11800 Praha 1, Czechoslovakia \\ valtr@copguk11.bidnet \\ and \\ Institut für Informatik, Freie Universität Berlin, \\ Arnimallee 2-6, 1000 Berlin 33, Federal Republic of Germany \\ valtr@math.fu-berlin.de
}

\begin{abstract}
For a finite set $A$ of points in the plane, let $q(A)$ denote the ratio of the maximum distance of any pair of points of $A$ to the minimum distance of any pair of points of $A$. For $k>0$ let $c_{\alpha}(k)$ denote the largest integer $c$ such that any set $A$ of $k$ points in general position in the plane, satisfying $q(A)<\alpha \sqrt{k}$ for fixed $\alpha \geq \sqrt{2 \sqrt{3} / \pi} \doteq 1.05$, contains at least $c$ convex independent points. We determine the exact asymptotic behavior of $c_{\alpha}(k)$, proving that there are two positive constants $\beta=\beta(\alpha), \gamma$ such that $\beta k^{1 / 3} \leq c_{\alpha}(k) \leq \gamma k^{1 / 3}$. To establish the upper bound of $c_{\alpha}(k)$ we construct a set, which also solves (affirmatively) the problem of Alon et al. [1] about the existence of a set $A$ of $k$ points in general position without a 7 -hole (i.e., vertices of a convex 7-gon containing no other points from $A$ ), satisfying $q(A)<\alpha \sqrt{k}$. The construction uses "Horton sets," which generalize sets without 7-holes constructed by Horton and which have some interesting properties.
\end{abstract}

\section{Introduction}

All points in this paper lie in the plane. Points $x_{1}, x_{2}, \ldots, x_{n}$ are convex independent, if they are vertices of a convex $n$-gon. Convex independent points $x_{1}, x_{2}, \ldots, x_{n} \in A$ form an $n$-hole in a set $A$ of points in the plane, if they form a convex $n$-gon containing no other points from $A$. For any positive integer $n$, let $f(n)$ be the smallest integer such that every set of $f(n)$ points in general position in the plane (i.e., no three lie on a line), contains $n$ convex independent points. Erd6s and 
Szekeres [2], [3] proved that $f(n)$ exists and showed that

$$
2^{n-2}+1 \leq f(n) \leq\left(\begin{array}{c}
2 n-4 \\
n-2
\end{array}\right)+1 .
$$

Alon et al. [1] studied the effect of restricting the geometry of the configurations. The configurations which establish the lower bound on $f(n)$ have some pairs of points very close together and others very far apart. The authors of [1] investigated the effect of placing an upper bound on the ratio of the maximum and minimum distances and showed that in this case substantially stronger results are true.

Let $A$ be a set of $k$ points in general position in the plane. We define

$$
q(A)=\frac{\max \{d(a, b): a, b \in A, a \neq b\}}{\min \{d(a, b): a, b \in A, a \neq b\}},
$$

where $d(a, b)$ is the Euclidean distance of two points $a$ and $b$. We can assume without loss of generality that $\min \{d(a, b): a, b \in A, a \neq b\}=1$.

In this case $q(A)$ is the diameter of $A$. It is interesting to study the asymptotic behavior of the smallest value of the maximum number of convex independent points in a set $A$ of $k$ points in general position, which satisfies an inequality $q(A)<\alpha \sqrt{k}$, for some positive constant $\alpha$. Let us note that there exists a positive constant $\alpha_{0}$ such that the inequality $q(A) \geq \alpha_{0} \sqrt{k}$ holds for any number $k$ and for any set $A$ of $k$ points in the plane (see [1]). Alon et al. [1] proved that if $q(A)<\alpha \sqrt{k}$, then the set $A$ contains at least $\beta k^{1 / 4}$ convex independent points for some positive constant $\beta=\beta(\alpha)$. In Section 2 we prove by probabilistic methods a stronger result: If $q(A)<\alpha \sqrt{k}$, then the set $A$ contains at least $\beta k^{1 / 3}$ convex independent points for some positive constant $\beta=\beta(\alpha)$.

On the other hand, Alon et al. [1] showed by probabilistic arguments that, for every fixed $\varepsilon>0$, the following holds: For any large integer $k>k(\varepsilon)$ there is a set $A$ of $k$ points in the plane satisfying $q(A)<4 \sqrt{k}$, which does not contain more than $k^{1 / 3+\varepsilon}$ convex independent points. In Section 4 we show a stronger result. For any constant $c>4 \cdot(2 / \pi)^{1 / 3} \sqrt{3} \doteq 5.96$ and for any large enough integer $k>k(c)$, we construct a set $A_{k}$ of $k$ points in general position in the plane satisfying $q\left(A_{k}\right)<\alpha \sqrt{k}$ for $\alpha \geq \sqrt{2 \sqrt{3} / \pi} \doteq 1.05$, which does not contain more than $c k^{1 / 3}$ convex independent points. Note that for every $\alpha<\sqrt{2 \sqrt{3} / \pi}$ there is a set $A$ of $k$ points which satisfies $q(A)<\alpha \sqrt{k}$ only for small $k$ (it is proved in Section 4 ).

In Section 4 we also study the existence of 7 -holes in a set $A$ of $k$ points satisfying $q(A)<\alpha \sqrt{k}$. Horton [5] constructed, for every $k$, a set $A$ of $k$ points in general position without 7 -holes. We prove that the set $A_{k}$ (mentioned above) also does not contain 7-holes. It solves for any constant $\alpha$ the problem from [1] whether, for large $k$, there exists a set $A$ of $k$ points satisfying $q(A)<\alpha \sqrt{k}$, which does not contain 7-holes. Indeed, as we say above, for every $\alpha<\sqrt{2 \sqrt{3 / \pi}}$ there is a set $A$ of $k$ points satisfying $q(A)<\alpha \sqrt{k}$ only for small $k$. 
Section 3 gives the definition and some properties of "Horton sets." Horton sets are "anticonvex" sets whose definition is based on ideas of Horton [5]. (By "anticonvex" sets we mean sets with no large holes and no large convex independent subsets.) These sets are used in constructions in Section 4.

\section{The Lower Bound on $c_{\alpha}(k)$}

In this section we estimate the lower bound on $c_{x}(k)$ by proving Theorem 2.1 .

Theorem 2.1. For any positive constant $\alpha$, there is a positive constant $\beta=\beta(\alpha)$ such that any set $A$ of $k$ points in general position, satisfying $q(A)<\alpha \sqrt{k}$, contains at least $\beta k^{1 / 3}$ convex independent points.

Assume that $A$ is a set of $k$ points in general position such that $q(A)<\alpha \sqrt{k}$ and the minimum distance of a pair of points from $A$ is 1 . Then $q(A)$ is the diameter of $A$ and $A$ is placed inside a disk $D$ of radius $r=\alpha \sqrt{k}$ centered at a point $O \in A$.

Let us consider a circle $C$ of radius $r$ centered at a point $S$. We place $\left\lfloor k^{1 / 3}\right\rfloor$ rectangles with sides $s=\alpha k^{1 / 6}$ and $t=\alpha k^{-1 / 6}$ so that they touch the circle $C$ from inside in regular intervals and one of the two longer sides of each rectangle is a chord of the circle $C$ (see Fig. 1). Traveling counterclockwise along the circle $C$, we denote the touching rectangles by $R_{1}, R_{2}, \ldots, R_{\left\lfloor k^{1}\right.}$ and their centers by $S_{1}$, $S_{2}, \ldots, S_{\left[k^{12}\right]}$ (in the order of their appearance along the circle).

Lemma 2.2. If we choose at most one point from each of the rectangles $R_{i}$, $i=1, \ldots,\left\lfloor k^{1 / 3}\right\rfloor$, then the chosen points are convex independent.

Proof. In the proof we use the points $K, L, L^{\prime}, L^{\prime \prime}, M, M^{\prime}$, and $N$, whose definition is apparent from Fig. 2. For any $i=1,2, \ldots,\left\lfloor k^{1 / 3}\right\rfloor$, we need to prove that the rectangle $R_{i}$ lies entirely on the other side of the straight line $L L^{\prime \prime}$ than the rectangles $R_{i-1}$ and $R_{i+1}$. In other words, we need to prove $\left|L^{\prime} N\right|>t+\left|M^{\prime} N\right|$.

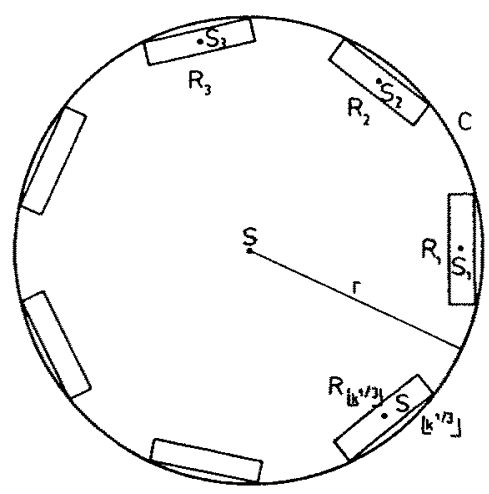

Fig. 1 

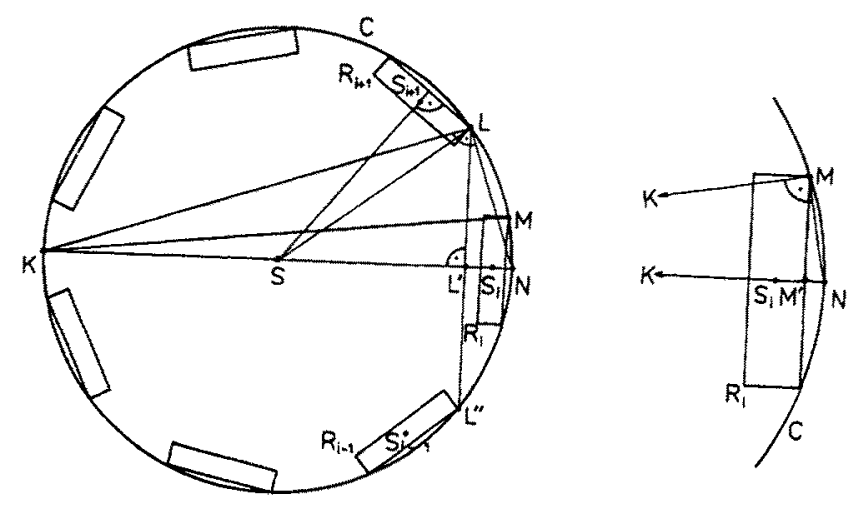

Fig. 2

We use basic claims about rectangular triangles and estimate:

$$
\begin{aligned}
\measuredangle L S L^{\prime} & =\measuredangle S_{i+1} S L^{\prime}-\measuredangle S_{i+1} S L=\frac{2 \pi}{\left\lfloor k^{1 / 3}\right\rfloor}-\arcsin \frac{s / 2}{r} \\
& \geq \frac{2 \pi}{k^{1 / 3}}-\frac{\pi}{2} \cdot \frac{s / 2}{r}=\frac{7 \pi}{4} k^{-1 / 3} \\
\left|L L^{\prime}\right| & =|S L| \cdot \sin \left(\Varangle L S L^{\prime}\right) \geq|S L| \cdot \frac{2}{\pi} \cdot \Varangle L S L^{\prime} \\
& \geq \alpha \sqrt{k} \cdot \frac{2}{\pi} \cdot \frac{7 \pi}{4} k^{-1 / 3}=\frac{7}{2} \alpha k^{1 / 6}, \\
\left|L{ }^{\prime} N\right|-\left|M^{\prime} N\right| & =\frac{\left|L L^{\prime}\right|^{2}}{\left|K L^{\prime}\right|}-\frac{\left|M M^{\prime}\right|^{2}}{\left|K M^{\prime}\right|}>\frac{\left|L L^{\prime}\right|^{2}-\left|M M^{\prime}\right|^{2}}{\left|K M^{\prime}\right|}>\frac{\left(\frac{7}{2} \alpha k^{1 / 6}\right)^{2}-\left(\frac{1}{2} \alpha k^{1 / 6}\right)^{2}}{2 \alpha \sqrt{k}} \\
& =6 \alpha k^{-1 / 6}=6 t .
\end{aligned}
$$

Thus $\left|L^{\prime} N\right|-\left|M^{\prime} N\right|>t$.

So if at least $\beta k^{1 / 3}$ of the rectangles $R_{i}$ are nonempty (i.e., contain at least one point of $A$ ), then at least $\beta k^{1 / 3}$ points of $A$ are convex independent. Therefore, for a fixed set $A$, we look for a placement of the circle $C$ and the rectangles $R_{i}$ in the plane such that at least $\beta k^{1 / 3}$ of the rectangles $R_{i}$ are nonempty. We proceed by probabilistic methods.

Let $M$ be the set of all rectangles $R$ with sides $s$ and $t$ and with center in the disk $D^{\prime}$ of radius $3 r$ centered at the point $O$. For any rectangle $R$ from $M$, the direction of the sides of length $s$ is called the direction of the rectangle $R$. Since $M$ is equivalent to the product of $D^{\prime}$ (corresponding to the placement of the center of $R)$ and $\langle 0, \pi)$ (corresponding to the direction of $R$ ), there is a measure $\mu$ on $M$ which is equivalent to the product of Lebesgue measures on $D^{\prime}$ and on $\langle 0, \pi)$. 
Here is a key lemma:

Lemma 2.3. There is a positive constant $c=c(\alpha)$ such that

$$
\mu(\{R \in M: R \cap A \neq \varnothing\}) \geq c \mu(M)
$$

The proof of Lemma 2.3 relies on two lemmas.

Lemma 2.4. Let $a$ be a point from $A$ and let $A(j)$ be the set of points $a^{\prime}$ from $A$ such that $j \leq d\left(a, a^{\prime}\right)<j+1$, where $d\left(a, a^{\prime}\right)$ is Euclidean distance of points a and $a^{\prime}$. For every positive integer $j$ the set $A(j)$ contains at most $c_{1} j$ points, for some positive constant $c_{1}$.

Proof. Consider the $15 j$ straight lines of directions $(i / 15 j) \pi, i=0,1, \ldots, 15 j-1$, going through the point $a$. Together with the circle of radius $\left(j+\frac{1}{2}\right)$ centered at $a$ they divide the plane into $60 j$ parts. Assume two points $b, c \in A(j)$ lie in the same part. Then

$$
d(b, c) \leq(\measuredangle b a c) \cdot d(a, b)+|d(a, b)-d(a, c)| \leq \frac{\pi}{15 j}(j+1)+\frac{1}{2}<1 .
$$

Hence each of the $60 j$ parts contains at most one point from $A(j)$, and setting $c_{1}=60$ gives the result.

For $a \in A, i=0,1, \ldots, k$, we denote:

$$
\begin{aligned}
M(a) & =\{R \in M: a \in R\}, \\
M_{i} & =\{R \in M:|R \cap A|=i\}, \\
m_{i} & =\mu\left(M_{i}\right) .
\end{aligned}
$$

Lemma 2.5. There are two positive constants $c_{2}$ and $c_{3}$ such that:

(i) $\forall a \in A: \mu(M(a))=c_{2}$,

(ii) $\forall a \in A: \sum_{a^{\prime} \in A} \mu\left(M(a) \cap M\left(a^{\prime}\right)\right) \leq c_{3}$.

Proof. (i) Centers of rectangles $R \in M(a)$ with fixed direction $\varepsilon \in\langle 0, \pi)$ lie in a rectangle with a center $a$, sides s and $t$, and direction $\varepsilon$. Thus, $\mu(M(a))=\pi \cdot s t=\pi \alpha^{2}$.

(ii) Directions of rectangles from $M(a) \cap M\left(a^{\prime}\right)$ are in an interval $I$ of length $2 \arcsin \left(t / d\left(a, a^{\prime}\right)\right.$ ) (if $t<d\left(a, a^{\prime}\right)$ ) or $\pi$ (if $t \geq d\left(a, a^{\prime}\right)$ ). In both cases the length of the interval $I$ is at most $\pi t / d\left(a, a^{\prime}\right)$. Since the rectangles from $M(a) \cap M\left(a^{\prime}\right)$ lie in $M(a)$,

$$
\mu\left(M(a) \cap M\left(a^{\prime}\right)\right) \leq|I| \cdot s t \leq \frac{\pi t}{d\left(a, a^{\prime}\right)} \cdot \alpha^{2} \leq c_{4} \frac{k^{-1 / 6}}{\left\lfloor d\left(a, a^{\prime}\right)\right\rfloor}
$$

for some positive constant $c_{4}$. 
If the distance $d\left(a, a^{\prime}\right)$ is greater than $2 s \geq \sqrt{s^{2}+t^{2}}$, then $M(a) \cap M\left(a^{\prime}\right)$ is empty. Hence

$$
\begin{aligned}
\sum_{a^{\prime} \in A} \mu\left(M(a) \cap M\left(a^{\prime}\right)\right) & =\mu(M(a))+\sum_{j=1}^{\lfloor 2 s\rfloor} \sum_{a^{\prime} \in A(j)} \mu\left(M(a) \cap M\left(a^{\prime}\right)\right) \\
& \leq c_{2}+\sum_{j=1}^{\lfloor 2 s\rfloor} \sum_{a^{\prime} \in A(j)} c_{4} \frac{k^{-1 / 6}}{j} \leq c_{2}+\sum_{j=1}^{\lfloor 2 s\rfloor} c_{1} j \cdot c_{4} \frac{k^{-1 / 6}}{j} \leq c_{3}
\end{aligned}
$$

for some positive constant $c_{3}$.

Proof of Lemma 2.3. For the sums $\sum_{i=1}^{k} m_{i}, \sum_{i=1}^{k} t^{2} m_{i}$, the Cauchy-Buniakowski inequality (a special case of the Hölder inequality) has the following form:

$$
\sum_{i=1}^{k} m_{i} \sum_{i=1}^{k} i^{2} m_{i} \geq\left(\sum_{i=1}^{k} i m_{i}\right)^{2}
$$

Now we estimate the sums $\sum_{i=1}^{k} i m_{i}, \sum_{i=1}^{k} i^{2} m_{i}$ :

$$
\begin{aligned}
& \sum_{i=1}^{k} i m_{i}=\sum_{a \in A} \mu(M(a))=\sum_{a \in A} c_{2}=c_{2} k \\
& \sum_{i=1}^{k} i^{2} m_{i}=\sum_{a \in A} \sum_{a^{\prime} \in A} \mu\left(M(a) \cap M\left(a^{\prime}\right)\right) \leq \sum_{a \in A} c_{3}=c_{3} k .
\end{aligned}
$$

We get

$$
\begin{gathered}
\sum_{i=1}^{k} m_{i} \geq \frac{\left(\sum_{i=1}^{k} i m_{i}\right)^{2}}{\sum_{i=1}^{k} i^{2} m_{i}} \geq \frac{\left(c_{2} k\right)^{2}}{c_{3} k}=\frac{c_{2}^{2}}{c_{3}} k, \\
\frac{\mu(\{R \in M: R \cap A \neq \varnothing\})}{\mu(M)}=\frac{\sum_{i=1}^{k} m_{i}}{\mu(M)} \geq \frac{\left(c_{2}^{2} / c_{3}\right) k}{\pi \cdot \pi(3 \alpha \sqrt{k})^{2}}=c>0
\end{gathered}
$$

for some positive constant $c$.

Proof of Theorem 2.1. Let the circle $C$ be randomly placed inside $D^{\prime}$, and let the collection of the rectangles $R_{i}, i=1, \ldots,\left\lfloor k^{1 / 3}\right\rfloor$, be randomly rotated inside $C$. So, for every $i=1, \ldots,\left\lfloor k^{1 / 3}\right\rfloor$, the rectangle $R_{i}$ is chosen from a set $S$, which contains nonempty rectangles from $M$ twice (see Fig. 3) and empty rectangles from $M$ at most twice. The rectangle $R_{i}$ is chosen from the set $S$ according to a probabilistic measure, which is a multiple of the measure $\mu$. Thus, the rectangle $R_{i}$ is nonempty with a probability

$$
\frac{\mu(\{R \in S: R \cap A \neq \varnothing\})}{\mu(S)} \geq \frac{\mu(\{R \in M: R \cap A \neq \varnothing\})}{\mu(M)} \geq c .
$$




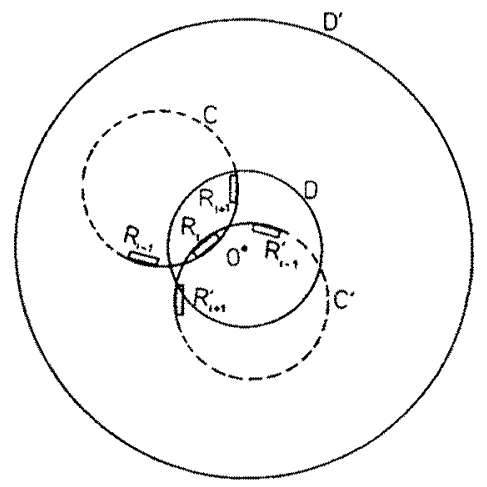

Fig. 3

The expected number of nonempty rectangles $R_{i}, i=1, \ldots,\left\lfloor k^{1 / 3}\right\rfloor$, is at least $c\left\lfloor k^{1 / 3}\right\rfloor \geq(c / 2) k^{1 / 3}$. Consequently, there is a placement of the rectangles $R_{i}$ such that at least $(c / 2) k^{1 / 3}$ rectangles $R_{i}$ are nonempty. Now, setting $\beta=c / 2$, Lemma 2.2 completes the proof of Theorem 2.1 .

The following theorem can be proved by similar arguments (if $r=\alpha k^{\tau}, s=\alpha k^{\tau / 3}$, $\left.t=\alpha k^{-\tau / 3}, i \in\left\{1,2, \ldots,\left\lfloor k^{2 \tau / 3}\right]\right\}\right)$.

Theorem 2.6. For any $\tau \in\left\langle\frac{1}{2}, \frac{3}{4}\right)$ and for any positive constant $\alpha$, there exists a positive constant $\beta=\beta(\alpha, \tau)$ such that any set $A$ of $k$ points in general position satisfying $q(A)<\alpha k^{\tau}$ contains at least $\beta k^{1-4 t / 3}$ convex independent points.

On the other hand, using the original construction of Erdbs and Szekeres [3], it is not difficult to obtain a set $S$ of $k$ points, which satisfies $q(S)<k$ and does not contain more than $\left(\log _{2} k+2\right)$ convex independent points.

\section{Horton Sets}

This section brings a definition of "Horton sets" and gives some other definitions and claims used in the next section.

If $a$ is a point, then its $x$-coordinate is denoted by $x(a)$. We say that $k$ points are in strongly general position if they are in general position and they have distinct $x$-coordinates. If $H$ is a set of $k$ points in strongly general position, then we usually use the notation

$$
H=\left\{h_{0}, h_{1}, \ldots, h_{k-1}\right\}, \quad x\left(h_{0}\right)<x\left(h_{1}\right)<\cdots<x\left(h_{k-1}\right) .
$$

A nonsingular linear transformation is a transformation

$$
T:[x, y] \mapsto[s x+t y, u x+v y],
$$

where $s, t, u, v$ are real numbers and

$$
\operatorname{det}\left|\begin{array}{ll}
s & t \\
u & v
\end{array}\right| \neq 0 .
$$


Observation 3.1. Let $A$ be a set of points in general position and let $T$ be a nonsingular linear transformation. Then points $p_{1}, \ldots, p_{n} \in A$ are convex independent or form an $n$-hole in $A$ if and only if points $T\left(p_{1}\right), \ldots, T\left(p_{n}\right) \in T(A)$ are convex independent or form an $n$-hole in $T(A)$, respectively.

A sequence of $r$ points $p_{1}, p_{2}, \ldots, p_{r}, x\left(p_{1}\right)<x\left(p_{2}\right)<\cdots<x\left(p_{r}\right)$ is said to be

(i) convex, if for all $i, j, k, 1 \leq i<j<k \leq r$, the point $p_{j}$ lies below the straight line $p_{i} p_{k}$

(ii) concave, if for all $i, j, k, 1 \leq i<j<k \leq r$, the point $p_{j}$ lies above the straight line $p_{i} p_{k}$.

A convex sequence of $r$ points $p_{1}, p_{2}, \ldots, p_{r}$ is upper closed by a point $p$, if $x\left(p_{1}\right)<x(p)<x\left(p_{r}\right)$ and the point $p$ lies above the polygonal line $p_{1} p_{2} \cdots p_{r}$. A concave sequence of $r$ points $p_{1}, p_{2}, \ldots, p_{r}$ is lower closed by a point $p$, if $x\left(p_{1}\right)<x(p)<x\left(p_{r}\right)$ and the point $p$ lies below the polygonal line $p_{1} p_{2} \cdots p_{r}$.

The set $A$ of points in strongly general position is said to be

(i) upper $r$-closed if every convex sequence of $r$ points from $A$ is upper closed by some point from $A$,

(ii) lower $r$-closed if every concave sequence of $r$ points from $A$ is lower closed by some point from $A$,

(iii) $r$-closed if it is both upper and lower $r$-closed.

Definition 3.2. Let $C$ and $D$ be two sets of points in strongly general position. If the set $C$ lies entirely below any straight line connecting pair of points from $D$ and the set $D$ lies entirely above any straight line connecting a pair of points from $C$, then we say that $C$ lies deep below $D$ and $D$ lies high above $C$.

Theorem 3.3. Let $A$ and $B$ be finite sets of points in strongly general position, which contain no $(r+s-1)$-hole. Let $A$ be upper $r$-closed, let $B$ be lower s-closed, and let $A$ lie deep below $B$. Then the set $A \cup B$ also contains no $(r+s-1)$-hole.

Proof. Let $H$ be an $(r+s-1)$-hole of the set $A \cup B$. We denote $A^{\prime}=H \cap A$, $B^{\prime}=H \cap B$. The sets $A^{\prime}$ and $B^{\prime}$ are nonempty because the sets $B$ and $A$ contain no $(r+s-1)$-holes. Since $A^{\prime}$ lies deep below $B^{\prime}$, the points of $A^{\prime}$ form a convex sequence, and the points of $B^{\prime}$ form a concave sequence. There are two possibilities: $A^{\prime}$ contains at least $r$ points or $B^{\prime}$ contains at least $s$ points. In the first case a point of $A$ which upper closes the convex sequence of points of $A^{\prime}$ lies in the convex hull of $H=A^{\prime} \cup B^{\prime}$. Similarly, in the second case a point of $B$ which lower closes the concave sequence of points of $B^{\prime}$ lies in the convex hull of $H=A^{\prime} \cup B^{\prime}$. This completes the proof.

Lemma 3.4. Let $C$ and $D$ be finite sets of points such that the points of $C \cup D$ are in strongly general position, and let $C$ lie deep below $D$. Then if $a_{1}, a_{2}, \ldots, a_{n}$ is $a$ convex sequence of $n$ points from the set $C \cup D$, then either the points $a_{2}, a_{3}, \ldots, a_{n-1}$ lie in $C$ or all the points $a_{1}, a_{2}, \ldots, a_{n}$ lie in $D$. Similarly, if $a_{1}, a_{2}, \ldots, a_{n}$ is a concave 
sequence of $n$ points from the set $C \cup D$, then either the points $a_{2}, a_{3}, \ldots, a_{n-1}$ lie in $D$ or all the points $a_{1}, a_{2}, \ldots, a_{n}$ lie in $C$.

Proof. Let the sequence $a_{1}, a_{2}, \ldots, a_{n}$ be convex. For any $i=2,3, \ldots, n-1$, if the point $a_{i}$ lies in $D$, then $D$ also contains the points $a_{i-1}$ and $a_{i+1}$. Thus if $D$ contains some of the points $a_{2}, a_{3}, \ldots, a_{n-1}$, then all points $a_{1}, a_{2}, \ldots, a_{n}$ lie in $D$. The case of a concave sequence $a_{1}, a_{2}, \ldots, a_{n}$ is similar.

For any finite set of $k$ points in strongly general position $H=\left\{h_{0}, h_{1}, \ldots, h_{k-1}\right\}$, $x\left(h_{0}\right)<x\left(h_{1}\right)<\cdots<x\left(h_{k-1}\right)$, we define subsets $H_{z, n}, 0 \leq z<n$ as follows:

$$
H_{z, n}=\left\{h_{i} \in H: i \equiv z(\bmod n)\right\}
$$

Now we define Horton sets and say something about them.

Definition 3.5. A finite set of points in strongly general position is called Horton if it is Horton according to a finite number of applications of the following rules:

(i) Every one-point set is Horton.

(ii) If the sets $H_{0,2}$ and $H_{1,2}$ are Horton and $H_{0,2}$ lies deep below or high above $H_{1,2}$, then the set $H$ is Horton.

Example 3.6. Horton [5] constructed, for any positive integer $t$, a set of $2^{t}$ points in general position without 7-holes as follows:

$$
S_{t}=\left\{[i, d(i)]: i=0,1, \ldots, 2^{t}-1\right\}
$$

where $d(i)=\sum_{j=1}^{t} a_{j} \cdot\left(2^{t}+1\right)^{j-1}$ and $\left(a_{1} a_{2} \cdots a_{t}\right)$ is the binary expansion of the integer $i$ (i.e., $\sum_{j=1}^{t} a_{j} \cdot 2^{t-j}=i$ and $a_{1}, a_{2}, \ldots, a_{t} \in\{0,1\}$ ). The sets $S_{t}$ are Horton sets according to our definition because their constructions use the following observation: If a set $H$ is a Horton set, then the set $H \cup(H+(\varepsilon, M))$ is also a Horton set, where $(H+(\varepsilon, M))$ is the set $H$ shifted by a vector $(\varepsilon, M)$ with sufficiently small $x$-coordinate $\varepsilon>0$ and sufficiently large $y$-coordinate $M>0$. (Indeed, the sets $(H \cup(H+(\varepsilon, M)))_{0,2}=H$ and $(H \cup(H+(\varepsilon, M)))_{1,2}=H+(\varepsilon, M)$ are Horton, and the first one lies deep below the second one.)

For any Horton set $H$, we denote the sets $H_{0,2}$ and $H_{1,2}$ by $H_{\text {low }}$ and $H_{\text {upp }}$ in such order that $H_{\text {low }}$ lies deep below $H_{\text {upp }}$.

Observation 3.7. Any contiguous segment of a Horton set $H$, i.e., $H^{\prime}=$ $\left\{h_{i} \in H: i_{0} \leq i \leq i_{1}\right\}$, is a Horton set.

Proof. We prove the observation by induction. For every one-point set the observation holds. Let $H$ be a Horton set and let the observation hold for the sets $H_{\text {low }}$ and $H_{\text {upp }}$ (i.e., for the sets $H_{0,2}$ and $H_{1,2}$ ) whose sizes are less than $|H|$. Assume $H^{\prime}$ is a contiguous segment of $H$. Since the sets $H^{\prime} \cap H_{\text {low }}$ and $H^{\prime} \cap H_{\text {upp }}$ 
are contiguous segments of $H_{\text {low }}$ and $H_{\text {upp }}$, respectively, they are both Horton. $H^{\prime} \cap H_{\text {low }}$ lies deep below $H^{\prime} \cap H_{\text {upp }}$. The sets $H^{\prime} \cap H_{\text {low }}$ and $H^{\prime} \cap H_{\text {upp }}$ are equal to the sets $H_{0,2}^{\prime}$ and $H_{1,2}^{\prime}$ in some order. Hence the set $H^{\prime}$ is Horton.

Now we prove two theorems which show that Horton sets are a good example of "anticonvex" sets.

Theorem 3.8. Every Horton set is 4-closed and contains no 7-hole.

Theorem 3.9. Let a Horton set $H$ contain a convex or a concave sequence of length $t$. If $t$ is odd, then $H$ contains at least $h(t)=2^{(t+1) / 2}-1$ points. If $t$ is even, then $H$ contains at least $h(t)=3 \cdot 2^{t / 2-1}-1$ points.

Proof of Theorem 3.8. We use the induction. Theorem 3.8 holds for every one-point set. Let $H$ be a Horton set and let $H_{\text {low }}$ and $H_{\text {upp }}$ be 4-closed sets with no 7-holes. The set $H=H_{\text {low }} \cup H_{\text {upp }}$ contains no 7-hole (Theorem 3.3). Consider a convex sequence $a, b, c, d$ of length 4 in the set $H$. According to Lemma 3.4 either all $a, b, c, d$ lie in $H_{\text {upp }}$, or both $b$ and $c$ lie in $H_{\text {low }}$. In the first case the sequence $a, b, c, d$ is upper closed by some point from $H_{\mathrm{upp}}$. In the second case, if $b=h_{i}$, the point $h_{i+1} \in H_{\text {upp }}$ upper closes the sequence $a, b, c, d$. Hence the set $H$ is upper 4-closed and similarly also lower 4-closed.

Proof of Theorem 3.9. Values $h(1)=1$ and $h(2)=2$ are obviously correct. Assume a Horton set $H$ contains a convex or a concave sequence $a_{1}, a_{2}, \ldots, a_{t+2}$ of length $(t+2)$. According to Lemma 3.4 the points $a_{2}, a_{3}, \ldots, a_{t+1}$ lie either all in $H_{\text {low }}$

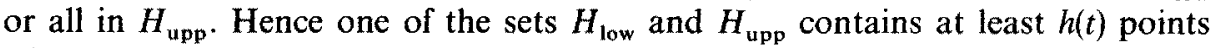
whose $x$-coordinates are in the interval $\left\langle x\left(a_{2}\right), x\left(a_{t+1}\right)\right\rangle$. Since points from $H$ are alternately from the subsets $H_{\text {low }}$ and $H_{\mathrm{upp}}$, and $a_{1}$ and $a_{t+2}$ both lie outside the interval $\left\langle x\left(a_{2}\right), x\left(a_{t+1}\right)\right\rangle$, the set $H$ contains at least $2 h(t)+1=h(t+2)$ points.

Claim 3.10 is often used in Section 4.

Claim 3.10. For every Horton set $H$, all subsets $H_{z, n}, 0 \leq z<n$, are Horton.

Proof. The proof is in two steps:

(i) Claim 3.10 holds for every odd $n$,

(ii) if Claim 3.10 holds for an integer $n$, then it holds also for an integer $n^{\prime}=2 n$.

(i) For a fixed odd $n$ we prove Claim 3.10 by induction on the size of $H$. Claim 3.10 holds for any one-point Horton set. Assume $H$ is a Horton set and Claim 3.10 holds for $n$ and the Horton sets $H_{0,2}$ and $H_{1,2}$. For any integer $z, 0 \leq z<n$, we denote $A=\left(H_{z, n}\right)_{0,2}, B=\left(H_{z, n}\right)_{1,2}$. If $z$ is even, then $A \subset H_{0,2}, B \subset H_{1,2}$, $A=\left(H_{0,2}\right)_{z / 2, n}$, and $B=\left(H_{1,2}\right)_{(n+z-1) / 2, n}$. If $z$ is odd, then $A \subset H_{1,2}, B \subset H_{0,2}$, $A=\left(H_{1,2}\right)_{(z-1) / 2, n}$, and $B=\left(H_{0,2}\right)_{(n+z) / 2, n}$. In both cases, $A$ lies deep below or high above $B$, and both $A$ and $B$ are Horton according to the inductive assumption.

(ii) Assume Claim 3.10 holds for a number $n$ and let $H$ be a Horton set. Let 
$0 \leq z<n$. If $z$ is even, then $H_{z, 2 n}=\left(H_{0,2}\right)_{z / 2, n}$. If $z$ is odd, then $H_{z, 2 n}=$ $\left(H_{1,2}\right)_{(z-1) / 2, n}$. In both cases, the set $H_{z, 2 n}$ is Horton according to the assumption. Claim 3.10 holds for $n^{\prime}=2 n$.

In the construction in Section 4 we use Horton sets with special location of points.

Lemma 3.11. For every $\varepsilon_{0}>0$ and for every positive integer $m$, we can construct a Horton set $H$ of $m$ points such that, for any $i=0,1, \ldots, m-1$, the point $h_{i}$ lies in the open $\varepsilon_{0}$-neighborhood of $x$-axis and its $x$-coordinate is equal to $i$.

Proof. Let $S$ be the contigous segment of the Horton set $S_{\left\lceil\log _{2} m\right\rceil}$ (see Example 3.6) which contains $m$ points with the smallest $x$-coordinates. Horton set $\Gamma(S)$ satisfies all the required conditions, where $T$ is a nonsingular linear transformation $T:[x, y] \mapsto[x, \delta y](\delta>0$ sufficiently small).

\section{The Upper Bound on $c_{\alpha}(k)$}

In this section we construct, for any positive integer $k$, the set $A_{k}$ for which Theorem 4.1 holds.

Theorem 4.1. For every $k$ and for every $c>4(2 / \pi)^{1 / 3} \sqrt{3} \doteq 5.96$, there is a set $A_{k}$ of $k$ points in general position satisfying $q\left(A_{k}\right)<\sqrt{2 \sqrt{3} / \pi} \sqrt{k}$, which contains neither a 7-hole nor, for large $k \geq k(c)$, more than $c k^{1 / 3}$ convex independent points.

First we construct, for every $\delta \in\left(0, \frac{1}{2}\right)$ and for every positive integer $m$, three auxiliary sets $L, B$, and $A$ having $m^{2}$ points. $L$ is the square lattice $m \times m, A$ has similar features as the set $A_{k}$ (where $k \sim$ const $\cdot m^{2}$ ) and $B$ is an intermediate step between $L$ and $A$. Both $A$ and $B$ form approximately the square lattice $m \times m$.

Consider two small numbers $\varepsilon, \varepsilon^{\prime} \in(0, \delta / 2)$, whose values will be specified later. We use a Horton set $H=\left\{h_{0}, h_{1}, \ldots, h_{m-1}\right\}$, which satisfies Lemma 3.11 for $\varepsilon_{0}=\varepsilon$, and a set $G=\left\{g_{0}, g_{1}, \ldots, g_{m-1}\right\}$, which satisfies Lemma 3.11 for $\varepsilon_{0}=\varepsilon^{\prime}$ after the interchange of axes (i.e., after the nonsingular linear transformation $T:[x, y] \mapsto$ $[y, x])$. We define:

$$
\begin{aligned}
& L=\left\{l_{i j}: i, j=0,1, \ldots, m-1\right\}, \\
& B=\left\{b_{i j}: i, j=0,1, \ldots, m-1\right\}, \\
& A=\left\{a_{i j}: i, j=0,1, \ldots, m-1\right\},
\end{aligned}
$$

where, for every $i, j=0,1, \ldots, m-1$,

$$
\begin{aligned}
l_{i j} & =[i, j], \\
b_{i j} & =h_{i}+(0, j), \\
a_{i j} & =h_{i}+\left(g_{j}-[0,0]\right)=g_{j}+\left(h_{i}-[0,0]\right) .
\end{aligned}
$$


For any fixed $i, j=0,1, \ldots, m-1$ we say that the points $l_{i j} \in L, b_{i j} \in B, a_{i j} \in A$ correspond to one another. Each point $b_{i j}$ from $B$ lies in the $\varepsilon$-neighborhood of the corresponding lattice point $l_{i j}$. Each point $a_{i j}$ from $A$ lies in the $\varepsilon^{\prime}$-neighborhood of the corresponding point $b_{i j}$ and in the $\delta$-neighborhood of the corresponding lattice point $l_{i j}$.

We prove that a suitable choice of $\varepsilon$ and $\varepsilon^{\prime}$ gives an "anticonvexity" of $A$ described by Claim 4.2 .

Claim 4.2. The set $A$ contains neither a 7 -hole nor, for $c^{\prime}>12^{2 / 3}=5.24$ and large $m \geq m_{0}\left(c^{\prime}\right)$, more than $c^{\prime}(m-4)^{2 / 3}$ convex independent points.

We determine $\varepsilon$ as an arbitrary positive number smaller than half of the smallest nonzero distance between a point from $L$ and a straight line connecting two points from $L$. Using such fixed $\varepsilon$ we construct the set $B$. Further we determine $\varepsilon^{\prime}$ as an arbitrary positive number smaller than half of the smallest nonzero distance between a point from $B$ and a straight line connecting two points from $B$. Using such fixed $\varepsilon^{\prime}$ we construct the set $A$.

Suppose that a point $l_{i j} \in L$ lies inside the convex hull of points $l_{i(1) j(1)}, \ldots$, $l_{i(r) j(r)} \in L$. The above choice of $\varepsilon$ ensures that the point $b_{i j} \in B$ lies inside the convex hull of the points $b_{i(1) j(1)}, \ldots, b_{i(r) j(r)} \in B$. Further, the above choice of $\varepsilon^{\prime}$ ensures that the point $a_{i j} \in A$ lies inside the convex hull of points $a_{i(1) j(1)}, \ldots, a_{i(r) j(r)} \in A$. This proves the following observation.

\section{Observation 4.3.}

(i) If seven points from $A$ form a 7-hole, then there is no point from $L$ inside the convex hull of the corresponding seven points from $L$.

(ii) If some points from $A$ are convex independent, then the corresponding points from $L$ lie on a perimeter of their convex hull.

The following claims together with Observation 4.3(i) prove that $A$ contains no 7-hole.

Claim 4.4. If seven points from $L$ are such that there is no point from $L$ inside their convex hull, then they lie on a straight line or on a pair of parallel lines.

Claim 4.5. If seven points from $L$ lie on a straight line or on a pair of parallel lines, then the corresponding seven points from $A$ do not form a 7-hole.

Proof of Claim 4.4. We show that every seven points of the lattice $Z \times Z$ (the set of all points with integer coordinates) with no points of $Z \times Z$ inside their convex hull lie on a straight line or on a pair of parallel lines. This is enough to prove claim 4.4 .

Consider seven lattice points which do not lie on a straight line. Let $a$ be a vertex of the convex hull of our seven points and let $b$ and $c$ be its adjacent vertices. Of course, the points $a, b$, and $c$ are among our seven lattice points. Let $b^{\prime}$ or $c^{\prime}$ be 


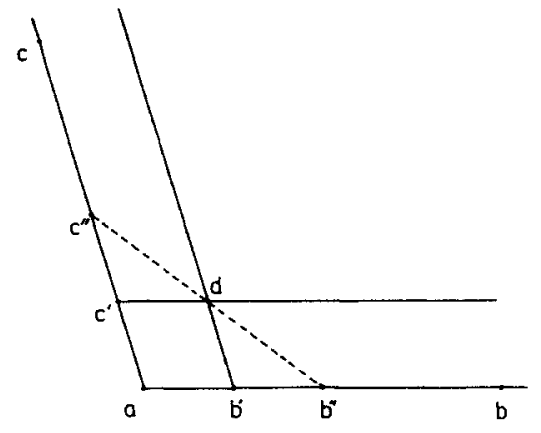

Fig. 4

the closest lattice point to $a$ on the line segment $a b$ or $a c$, respectively. We denote $b^{\prime \prime}=b^{\prime}+\left(b^{\prime}-a\right), c^{\prime \prime}=c^{\prime}+\left(c^{\prime}-a\right), d=b^{\prime}+\left(c^{\prime}-a\right)$ (see Fig. 4). The line segment $a b^{\prime \prime}$ contains only three lattice points $a, b^{\prime}, b^{\prime \prime}$, the line segment $a c^{\prime \prime}$ contains only three lattice points $a, c^{\prime}, c^{\prime \prime}$. No point inside the triangle $a b^{\prime} c^{\prime}$ is a lattice point because such points lie inside the triangle $a b c$ and, consequently, inside the convex hull of our seven lattice points. Also no point inside the triangle $b^{\prime} c^{\prime} d$ is a lattice point. Indeed, if a point $e$ inside the triangle $b^{\prime} c^{\prime} d$ is a lattice point, then a point $a+(d-e)$ inside the triangle $a b^{\prime} c^{\prime}$ is a lattice point. Similarly, there are no lattice points inside the line segments $b^{\prime} d$ and $c^{\prime} d$. If there is a lattice point inside the line segment $b^{\prime} c^{\prime}$, then $b=b^{\prime}, c=c^{\prime}$, and all our seven points lie on the straight line $b^{\prime} c^{\prime}$ and on its parallel line going through the point $a$. We finish the proof with the case that the parallelogram $a b^{\prime} d c^{\prime}$ contains no lattice points different from its vertices. In this case there are no lattice points between the parallel lines $a b^{\prime}$ and $c^{\prime} d$, and also between the parallel lines $a c^{\prime}$ and $b^{\prime} d$. Since the lattice point $d$ cannot lie inside the convex hull of our seven lattice points, they must lie on the semilines $a b^{\prime}, c^{\prime} d, a c^{\prime}$, and $b^{\prime} d$, and, consequently, either on the parallel semilines $a b^{\prime}$ and $c^{\prime} d$ or on the parallel semilines $a c^{\prime}$ and $b^{\prime} d$.

Before the proof of Claim 4.5 we present some notation and a lemma. A set of all points from $L$ lying on a straight line $p$ is denoted by $L_{p}$. A set of points from $B$ or $A$ corresponding to points from $L_{p}$ is denoted by $B_{p}$ or $A_{p}$, respectively.

Lemma 4.6. If $p$ is parallel to the $y$-axis, then after the interchange of axes the set $A_{p}$ is Horton. If $p$ is not parallel to the $y$-axis, then the set $A_{p}$ is Horton.

Proof. If $p$ is parallel to the $y$-axis, then after the interchange of axes the set $A_{p}$ is Horton because it is a shift of a Horton set. If $p$ is not parallel to the $y$-axis, then

$$
B_{p}=\left\{b_{i_{1} j_{1}}, b_{i_{2} j_{2}}, \ldots, b_{i_{s} j_{s}}\right\}, \quad 0 \leq i_{1}<i_{2}<\cdots<i_{s} \leq m
$$

By the definition of $B_{p}$, there are two numbers $\Delta i>0, \Delta j$ such that $\Delta i=$ $i_{2}-i_{1}=i_{3}-i_{2}=\cdots=i_{s}-i_{s-1}$ and $\Delta j=j_{2}-j_{1}=j_{3}-j_{2}=\cdots=j_{s}-j_{s-1}$. A 
nonsingular linear transformation $T:[x, y] \mapsto\left[x, y+j_{1}+\left(x-i_{1}\right) \Delta j / \Delta i\right]$ maps the set $\left\{h_{i_{1}}, \ldots, h_{i_{s}}\right\}$ onto the set $B_{p}$. The set $\left\{h_{i_{1}}, \ldots, h_{i_{s}}\right\}$ is Horton because it is a contiguous segment of some Horton set $H_{z, \Delta_{r}}$. The above nonsingular linear transformation $T$ maps any Horton set onto a Horton set since it preserves $x$-coordinates. Thus $B_{p}$ is Horton. Now from the choice of $\varepsilon^{\prime}$ it follows that $A_{p}$ is also Horton.

Proof of Claim 4.5. Let $p$ and $q$ be two parallel lines. We prove Claim 4.5 by showing that the set $A_{p} \cup A_{q}$ contains no 7-hole. If the lines $p$ and $q$ are not parallel to the $y$-axis, then the Horton sets $A_{p}$ and $A_{q}$ are without a 7-hole and 4-closed. The numbers $\varepsilon$ and $\varepsilon^{\prime}$ are determined so that $A_{p}$ lies high above or deep below $A_{q}$. It follows from Theorem 3.3 that $A_{p} \cup A_{q}$ does not contain a 7-hole. If the lines $p$ and $q$ are parallel to the $y$-axis, then a similar consideration after the interchange of axes completes the proof.

The first part of Claim 4.2 ( $A$ contains no 7-hole) is proved. Now we give the two simple lemmas and finish the proof of Claim 4.2 .

Lemma 4.7. Let $g(t)$ be the sum of the first $(t-1)$ terms of the sequence $1,1,2,2$, $3, \ldots$. If a Horton set $H$ contains a convex or a concave sequence of length $t$, then the size of $H$ is greater than $g(t)$.

Proof. It is sufficient to prove that $g(t)<h(t)$, where $h(t)$ is as in Theorem 3.9:

$$
\begin{aligned}
g(1) & =0<1=h(1), \\
g(2) & =1<2=h(2), \\
& \cdots, \\
g(t+2) & =g(t)+\lfloor(t+1) / 2\rfloor+\lceil(t+1) / 2\rceil \\
& =g(t)+t+1<h(t)+t+1 \leq h(t)+h(t)+1=h(t+2) .
\end{aligned}
$$

By $L_{1}$-metric we mean the metric defined by

$$
d_{1}\left(\left[x_{1}, y_{1}\right],\left[x_{2}, y_{2}\right]\right)=\left|x_{2}-x_{1}\right|+\left|y_{2}-y_{1}\right|
$$

Further in this section all distances and lengths are considered in $L_{1}$-metric.

Lemma 4.8. A sum of lengths (in $L_{1}$-metric) of any $2(n+1)^{2}$ pairwise different nonzero vectors with integer coordinates is greater than $\frac{4}{3} n^{3}$.

Proof. The number of vectors of length $i$ (in $L_{1}$-metric) with integer coordinates is equal to $4 i$, and a sum of their lengths is equal to $4 i^{2}$. The number of vectors of length at most $n$ with integer coordinates is equal to $\sum_{i=1}^{n} 4 i=2 n(n+1)$. A sum of their lengths is equal to $\sum_{i=1}^{n} 4 i^{2}=\frac{2}{3} n(n+1)(2 n+1)$, which is the smallest sum of $2 n(n+1)$ pairwise different nonzero vectors with integer coordinates. This shows Lemma 4.8 . 
Proof of Claim 4.2. As we said above, Observation 4.3(i) and Claims 4.4 and 4.5 prove that $A$ contains no 7 -hole. It remains to prove that the set $A$ of $m^{2}$ points does not contain more than $c^{\prime}(m-4)^{2 / 3}$ convex independent points, for $c^{\prime}>12^{2 / 3}$ and large $m \geq m_{0}\left(c^{\prime}\right)$.

Let $A^{\prime}$ be a convex independent subset of $A$, and let $L^{\prime}$ be the set of the points from $L$ corresponding to the points of $A^{\prime}$. All points of $L^{\prime}$ lie on the perimeter of the convex hull of $L^{\prime}$ (Observation 4.3(ii)). First suppose that the points from $L^{\prime}$ do not lie on a line. Let $l_{1}, l_{2}, \ldots, l_{s}$ be the vertices of the convex hull of $L^{\prime}$ in the counterclockwise order. For any $i=1,2, \ldots, s$, we define a vector $w_{i}=l_{i+1}-l_{i}$ with integer coordinates (if $i=s$, then naturally $l_{i+1}=l_{1}$ ) and a vector $v_{i}$ as the smallest vector with integer coordinates such that there is a positive integer $c_{i}$ with $w_{i}=c_{i} v_{i}$. So, on the line segment $l_{i} l_{i+1}$ there are just points $l_{i}, l_{i}+v_{i}, \ldots, l_{i}+$ $c_{i} v_{i}=l_{i+1}$ from the lattice $L$.

Now we investigate, for $i=1,2, \ldots, s$, how many points $l_{i}, l_{i}+v_{i}, \ldots, l_{i}+$ $c_{i} v_{i}=l_{i+1}$ can be from $L^{\prime}$. Assume the straight line $p=l_{i} l_{i+1}$ is not parallel to the $y$-axis. By $H_{i}$ we denote the set of points of $A$ corresponding to the points $l_{i}$, $l_{i}+v_{i}, \ldots, l_{i}+c_{i}^{i} v_{i}=l_{i+1}$. The set $H_{i}$ is Horton because it is a contiguous segment of the Horton set $A_{p}$. The construction of the set $A$ ensures that the points from $H_{i} \cap A^{\prime}$ form a convex or a concave sequence in the Horton set $H_{i}$. The set $H_{i}$ contains $\left(c_{i}+1\right)$ points. Lemma 4.7 says that $c_{i}+1>g\left(r_{i}\right)$, where $r_{i}=\left|H_{i} \cap A^{\prime}\right|$. If the straight line $p=l_{i} l_{i+1}$ is parallel to the $y$-axis, then we proceed similarly (with the interchange of axes). Again, $c_{i}+1>g\left(r_{i}\right)$.

The perimeter of the convex hull of $L^{\prime}$ (in $L_{1}$-metric) is less than or equal to the perimeter of the square $\langle 0, m-1\rangle \times\langle 0, m-1\rangle$, which equals $4(m-1)$. In our notation

$$
\sum_{i=1}^{s}\left\|w_{i}\right\| \leq 4(m-1)
$$

On the other hand,

$$
\begin{aligned}
\left\|w_{i}\right\|=c_{i}\left\|v_{i}\right\| \geq g\left(r_{i}\right)\left\|v_{i}\right\|=\underbrace{(1+1+2+2+3+\cdots)}_{r_{i}-1}\left\|v_{i}\right\| \\
=(\underbrace{\left.\left\|v_{i}\right\|+\left\|v_{i}\right\|+\left\|2 v_{i}\right\|+\left\|2 v_{i}\right\|+\left\|3 v_{i}\right\|+\cdots\right)}_{r_{i}-1}, \\
\sum_{i=1}^{s}\left\|w_{i}\right\| \geq \sum_{i=1}^{\sum_{i=1}^{s}} \underbrace{\left(\left\|v_{i}\right\|+\left\|v_{i}\right\|+\left\|2 v_{i}\right\|+\left\|2 v_{i}\right\|+\left\|3 v_{i}\right\|+\cdots\right)}_{r_{i}-1} .
\end{aligned}
$$

Since any two vectors $v_{i}$ and $v_{j}$ have different directions, each of the vectors $v_{i}$, $2 v_{i}, \ldots$ is different from each of the vectors $v_{j}, 2 v_{j}, \ldots$ Thus any vector occurs at most twice on the right-hand side of (2). By Lemma 4.8., if the number $\sum_{i=1}^{s}\left(r_{i}-1\right)$ of the vectors on the right-hand side of (2) is at least $2 \cdot 2(n+1)^{2}$ for an integer $n$, 
then the sum of their lengths is greater than $2 \cdot \frac{4}{3} n^{3}$. Therefore, if we find an integer $n$ such that

$$
4(n+1)^{2} \leq \sum_{i=1}^{s}\left(r_{i}-1\right)<4(n+2)^{2}
$$

then

$$
\sum_{i=1}^{s}\left\|w_{i}\right\|>\frac{8}{3} n^{3}
$$

Now inequality (1) above gives

$$
\begin{aligned}
& \frac{8}{3} n^{3}<4(m-1), \\
& 8 n^{3}<12(m-1) \\
& 4 n^{2}<12^{2 / 3}(m-1)^{2 / 3} .
\end{aligned}
$$

If $c^{\prime}>12^{2 / 3}$, then, for large $m$,

$$
4(n+2)^{2}<c^{\prime}(m-1)^{2 / 3}
$$

For any $c^{\prime}>12^{2 / 3}$ and for any large $m \geq m_{0}\left(c^{\prime}\right)$, the following upper bound of a size of the set $A^{\prime}$ is established:

$$
\left|A^{\prime}\right|=\sum_{i=1}^{s}\left(r_{i}-1\right)<4(n+2)^{2}<c^{\prime}(m-1)^{2 / 3} .
$$

The case when the points of $L^{\prime}$ lie on a line can be treated similarly.

Proof of Theorem 4.1. For a given positive integer $k$ we find an integer $m$ such that $(\sqrt{3} \pi / 8)(m-4)^{2}<k \leq(\sqrt{3} \pi / 8)(m-3)^{2}$. The nonsingular linear transformation $T:[x, y] \mapsto[x+y / 2, \sqrt{3} y / 2]$ maps the lattice $L$ of $m^{2}$ points onto the set $T(L)$, which forms the triangle lattice on the rhombus $R$ with vertices $[0,0]$, $[m-1,0],\left[\frac{3}{2}(m-1),(\sqrt{3} / 2)(m-1)\right]$, and $\left[\frac{1}{2}(m-1),(\sqrt{3} / 2)(m-1)\right]$. The triangle with vertices $[t, t],[t+1, t],[t, t+1]$, where $t=\lfloor(m-1) / 2\rfloor$, is mapped by $T$ onto a triangle, which we denote by $\Delta$. The expected number of points from $T(L)$ lying in the interior of the disk of diameter $\sqrt{2 \sqrt{3} / \pi} \cdot k^{1 / 2}$ centered randomly in the triangle $\triangle$ is equal to $2 / \sqrt{3} \cdot(\pi / 4)\left(\sqrt{2 \sqrt{3}} / \pi \cdot k^{1 / 2}\right)^{2}=k$. Thus, there is a disk $D$ of diameter $\sqrt{2 \sqrt{3} / \pi} \cdot k^{1 / 2}$ containing at least $k$ points from $T(L)$ in the interior. If $\delta>0$ is sufficiently small in the above construction of the set $A$, then the disk $D$ also contains at least $k$ points from $T(A)$ in the interior. Some $k$ of these points (for example, the ones with the smallest $x$-coordinates) form the set $A_{k}$ which satisfies $q\left(A_{k}\right)<\sqrt{2 \sqrt{3} / \pi} \cdot k^{1 / 2}$, contains no 7 -hole, and does not contain more 
than $c^{\prime}(m-4)^{2 / 3} \leq c^{\prime}(8 / \sqrt{3} / \pi)^{1 / 3} k^{1 / 3}$ convex independent points, for $c^{\prime}>12^{2 / 3}$ and large $k$. It follows that $A_{k}$ does not contain more than $c k^{1 / 3}$ convex independent points, for every $c>12^{2 / 3}(8 / \sqrt{3} / \pi)^{1 / 3}=4(2 / \pi)^{1 / 3} \sqrt{3} \doteq 5.96$ and large $k \geq k(c)$.

Similarly, it is possible to prove the following theorem.

Theorem 4.9. Let $\delta \in\left(\frac{1}{2}, 1\right)$. For any positive real number $\alpha>0$ there is a positive constant $c=c(\alpha)$ such that, for any large $k$, there exists a set $A_{k}$ of $k$ points in general position which satisfies $q\left(A_{k}\right)<\alpha k^{\delta}$ and contains at most $c k^{2(1-\delta) / 3}$ independent points.

Proof. The proof is short, and some details are left to the reader.

For large $k$ and $m=(\alpha / 2) k^{\delta}$, consider the above set $A$ of $m^{2}$ points satisfying Claim 4.2. Define the set $A_{k}$ as the set of all points $p$ of $A$ with $x$-coordinate in the form

$$
x(p)=d+n \cdot \frac{\alpha}{2} k^{\left(5^{0}-2\right) / 3}
$$

where $d \in\left\langle 0,(2 / \alpha) k^{(1-\delta) / 3}\right\rangle$ and $n$ is an integer. Thus the points of $A_{k}$ lie in $k^{2(1-\delta) / 3}$ strips of width $(2 / \alpha) k^{(1-\delta) / 3}$ and $A_{k}$ has about $k$ points.

Let $C$ be the convex hull of a convex independent subset of $A_{k}$. Less than $c_{1} k^{(1-\delta) / 3}$ sides of $C$ lie in only one strip, and less than $c_{2} k^{(1-\delta) / 3}$ sides of $C$ intersect at least two strips, where $c_{1}$ and $c_{2}$ are the two positive constants independent of $k, A$, and $C$. This gives Theorem 4.9 .

We conjecture that the result of Theorem 4.9 is the best possible and, on the other hand, Theorem 2.6 can be improved.

Let us remark that the coefficient $\alpha=\sqrt{2 \sqrt{3} / \pi} \doteq 1.05$ in Theorem 4.1 is the smallest one which makes sense:

Proposition 4.10. For every $\alpha<\sqrt{2 \sqrt{3} / \pi}$ and large enough $k$, there is no set $A$ of $k$ points in the plane satisfying $q(A) \leq \alpha \sqrt{k}$.

A possible proof is based on two known claims.

Claim A. The disk of diameter $R$ has the largest area among all figures with the diameter $R$. Its area is $(\pi / 4) R^{2}$.

Claim B. Let d(r) be the maximum number of points that can be placed on a convex figure with area $P$ so that minimum distance between any pair of them is at least $1 / r$. Then

$$
\lim _{r \rightarrow \infty} \frac{d(r)}{r^{2}}=\frac{2 \sqrt{3}}{3} P
$$


Claim A is proved in [6], Claim B is one of the basic claims about the covering. We conclude this paper with a question: Suppose that $A$ is a set of $k$ points in general position satisfying $q(A) \leq \alpha \sqrt{k}$ for fixed $\alpha \geq \sqrt{2 \sqrt{3} / \pi}$ and $k$ is large. Does it follow that $A$ contains a 6-hole? In the general case it is an open problem whether any large set of points in general position contains a 6-hole. On the other hand, Harborth [4] proved that any set $A$ of at least 10 points in general position contains a 5-hole.

\section{Acknowledgments}

I would like to thank Jaroslav Nešetril for bringing this problem to my attention, and both Jaroslav Nešetřil and Jiři Matoušek for their consultations, suggestions, reviewing my solutions, and help with the work on this paper. I also thank the referee for helpful and detailed comments.

\section{References}

1. N. Alon, M. Katchalski, and W. R. Pulleyblank: The maximum size of a convex polygon in a restricted set of points in the plane, Discrete Comput. Geom. 4 (1989), 245-251.

2. P. Erd6s, G. Szekeres: A combinatorial problem in geometry, Compositio Math. 2(1935), 464-470.

3. P. Erd6s, G. Szekeres: On some extremum problems in elementary geometry, Ann. Univ. Sci. Budapest 3/4 (1960/61), 53-62.

4. H. Harborth: Konvexe Fünfecke in ebenen Punktmengen, Elem. Math. 33 (1978), 116-118.

5. J. D. Horton: Sets with no empty convex 7-gons, Canad. Math. Bull. 26 (1983), 482-484.

6. I. M. Jaglom, W. G. Boltjanski: Konvexe Figuren, VEB Deutschen Verlag der Wissenschaften, Berlin (1956), example 76b, p. 53.

Received October 17, 1989, and in revised form February 6, 1990, and February 1, 1991. 